\title{
3 Research Square \\ High Expression of LGR6 is A Poor Prognostic Factor In Esophageal Carcinoma
}

\section{Takehito Ehara}

First Department of Surgery

Takeshi Uehara ( $\square$ tuehara@shinshu-u.ac.jp )

Department of Laboratory Medicine

\section{Takahiro Yoshizawa}

First Department of Surgery

\section{Tomoyuki Nakajima}

Department of Laboratory Medicine

\section{Yasuhiro Kinugawa}

Department of Laboratory Medicine

\section{Shota Kobayashi}

Department of Laboratory Medicine

\section{Shiho Asaka}

Department of Laboratory Medicine

\section{Mai Iwaya}

Department of Laboratory Medicine

\section{Tadanobu Nagaya}

Department of Gastroenterology

\section{Yusuke Miyagawa}

First Department of Surgery

\section{Hiroyoshi Ota}

Department of Laboratory Medicine

\section{Yuji Soejima}

First Department of Surgery

\section{Research Article}

Keywords: leucine-rich repeat-containing G-protein-coupled receptor 6, esophageal squamous cell carcinoma, RNA in situ hybridization

Posted Date: December 1st, 2021

DOI: https://doi.org/10.21203/rs.3.rs-1087394/v1 
License: (c) (i) This work is licensed under a Creative Commons Attribution 4.0 International License. Read Full License 


\section{Abstract}

Background: Leucine-rich repeat-containing G-protein-coupled receptor 6 (LGR6) promotes carcinogenesis and progression in some cancer types. However, there are few reports of LGR6 expression in esophageal squamous cell carcinoma (ESCC). LGR6 expression and clinicopathological features in ESCC were investigated by RNAscope, a high-sensitivity RNA in situ hybridization method.

Methods: Appropriate tumors were selected from 41 cases of ESCC from which tissue microarrays were generated, and LGR6 expression was identified by RNAscope.

Results: Thirty-seven patients had LGR6 expression. High LGR6 expression was observed in 17 cases and low LGR6 expression in 24 cases. LGR6 expression was significantly higher in high histological grade ESCC than in low histological grade ESCC $(P=0.0023)$. ESCC patients who received neoadjuvant chemotherapy had significantly higher $L G R 6$ expression than those without neoadjuvant chemotherapy $(P=0.0109)$. Furthermore, high $L G R 6$ expression showed a poorer prognosis than low $L G R 6$ expression (log-rank test, $P=0.0365$ ).

Conclusions: LGR6 may be a prognostic factor and a potential new therapeutic target in ESCC.

\section{Background}

Leucine-rich repeat-containing G-protein-coupled receptor 6 (LGR6) belongs to the leucine-rich repeatcontaining subgroup of the G-protein-coupled 7-transmembrane protein superfamily [1]. LGR6 has homology to LGR4 and LGR5 [1], and is a stem cell marker for hair follicles [2], lung [3], and mammary gland [4]. LGR6 is involved in tissue development, regeneration, and injury repair.

LGR6 is also involved in cancer initiation and progression in lung [5], breast [4], and ovarian cancer [6].

Esophageal squamous cell carcinoma (ESCC) has a poor prognosis [7], and improvements are required in treatment outcome. To improve the survival rate of patients, the establishment of new biomarkers and targets is required. In this study, we analyzed the clinicopathological features of $L G R 6$ and examined the possibility of their application to the treatment of ESCC.

\section{Methods}

\section{Patients and materials}

We identified 56 ESCC cases who underwent surgical resection between 2010 and 2019 at Shinshu University Hospital, Matsumoto, Japan, and evaluated their clinicopathological features. Among these patients, cases with invasion deeper than the submucosa were selected. Stage IV cases were excluded as well as those that were negative for the positive control (housekeeping gene). A re-evaluation of the cases was undertaken by two pathologists (T.U. and M.I.), and 41 ESCC cases remained as candidates for 
analysis. This study was approved by the ethics committee of Shinshu University, Japan (approval no. 4088).

\section{Histopathology}

Paraffin blocks fixed with $8 \%$ formaldehyde containing sufficient tumor for analysis were prepared for hematoxylin and eosin (HE) staining and tissue microarray analysis by extracting a core with a diameter of $3 \mathrm{~mm}$ from each case.

\section{LGR6 RNA in situ hybridization}

LGR6 mRNA detection was performed using the RNAscope kit (Advanced Cell Diagnostics, Hayward, CA, USA), as described previously [8]. Additionally, a four-step evaluation method was used, as we previously reported [8]. LGR6 mRNA expression was categorized as low expression (grades $0,1+$, and $2+$ ) and high expression (3+ and 4+). We analyzed the relationship between LGR6 expression and the clinicopathological data and prognosis of patients with ESCC, particularly regarding overall survival (OS). We also evaluated LGR6 expression in non-cancerous tissues of the background mucosa of ESCC.

\section{Statistical analysis}

Fisher's exact test was applied to assess statistical significance. A $P$-value of $<0.05$ was considered statistically significant. The OS rates of ESCC patients were calculated using the Kaplan-Meier method, and differences were compared using the log-rank test. A $P$-value of $<0.05$ was considered to be statistically significant. Statistical analysis was performed using JMP version 13 (SAS Institute Japan, Tokyo, Japan).

\section{Results}

\section{LGR6 expression in normal esophageal mucosa and ESCC}

LGR6 expression was identified in the basal cell layer of non-tumorous regions. In ESCC patients, 37 of 39 cases showed $L G R 6$ expression. Among them, 17 cases were identified as having high LGR6 expression (Fig. 1A and 1B), while LGR6 expression was completely absent in two cases (Fig. 1D and 1E). LGR6 expression varied from diffuse to scattered patterns.

\section{Relationship between LGR6 expression and clinicopathological characteristics}

LGR6 expression and clinicopathological data are shown in Table 1. LGR6 expression was significantly higher in high histological grade ESCC than in low histological grade ESCC $(P=0.0023)$. ESCC patients with neoadjuvant chemotherapy had significantly higher LGR6 expression than ESCC patients without neoadjuvant chemotherapy $(P=0.0109)$. No significant differences were identified between high $L G R 6$ expression and low LGR6 expression regarding age, sex, vascular invasion, or TNM stage. 
Table 1

LGR6 expression and clinicopathological characteristics

\begin{tabular}{|c|c|c|c|c|}
\hline & & LGR6 expres: & & \\
\hline Factors & $\mathrm{n}$ & High $(n=17)$ & Low $(n=24)$ & $P$-value \\
\hline Age & & & & 0.0624 \\
\hline >71years & 19 & 11 & 8 & \\
\hline$\leq 70$ years & 22 & 6 & 16 & \\
\hline Sex & & & & 0.6786 \\
\hline Male & 34 & 15 & 19 & \\
\hline Female & 7 & 2 & 5 & \\
\hline Neoadjuvant chemotherapy & & & & 0.0109 \\
\hline Yes & 21 & 13 & 8 & \\
\hline No & 20 & 4 & 16 & \\
\hline Vascular invasion & & & & 1.000 \\
\hline Present & 21 & 9 & 12 & \\
\hline Absent & 20 & 8 & 12 & \\
\hline TIL & & & & 1.000 \\
\hline High & 20 & 8 & 12 & \\
\hline Low & 21 & 9 & 12 & \\
\hline Histological grade & & & & 0.0023 \\
\hline High & 31 & 17 & 14 & \\
\hline Low & 10 & 0 & 10 & \\
\hline TNM stage & & & & 1.0000 \\
\hline$|-I|$ & 20 & 8 & 12 & \\
\hline III & 21 & 9 & 12 & \\
\hline
\end{tabular}

\section{Prognostic value of LGR6 in ESCC}

The prognostic value of LGR6 expression in ESCC was analyzed by the Kaplan-Meier method and logrank test (Fig. 2). The median OS for the study patients was 537 (range; 269.5-2299.2) days. High LGR6 
expression [median OS: 322 (range; 175-555.5) days] was correlated with significantly worse OS than low LGR6 expression [median OS: 992 (range; 435.5-1825.5) days] (log-rank test, $P=0.0365$ ).

\section{Discussion}

This is the first report of RNAscope analysis of $L G R 6$ expression in the esophagus. The correlation of poor prognosis with high $L G R 6$ expression is consistent with previous immunohistochemical reports [9]. LGR6 expression is associated with ESCC differentiation in immunostaining studies [9]. However, maintenance of epithelial tissue is promoted by resident stem cells [10]. Given that $L G R 6$ is used as a stem cell marker in the epidermis [2], identification of the position of stem cells exhibiting LGR6 expression in the esophageal epithelium, which is the same stratified squamous epithelium in the skin, may lead to applications such as regenerative medicine.

In a murine study, it was reported that $L G R 6$ has the characteristics of cancer stem cells (CSCs) in squamous cell carcinoma of the skin [11]. LGR6 interacts with R-spondin 1-4 and is involved in the Wnt/ $\beta$ catenin signaling pathway via phosphorylation, exerting various biological actions $[9][12,13][14][15]$. The Wnt/ $\beta$-catenin signaling pathway plays an important role in the pathogenesis of various human diseases and tumors [13] LGR6 has been reported to promote the initiation and progression of cancers such as lung adenocarcinoma [5] and ovarian cancer including serous carcinoma [6]. LGR6 may contribute to the initiation and progression of ESCC by activating the Wnt/ $\beta$-catenin signaling pathway, but further research is needed to confirm this hypothesis.

High LGR6 expression in neoadjuvant chemotherapy cases indicated that LGR6-expressing cells may have CSC-like characteristics with chemoresistance. In fact, $L G R 6$ expression has also been reported to contribute to stem cell and chemoresistance in ovarian cancers, including serous carcinoma [6]. The function of chemoresistance is also considered to be influenced by the Wnt/ $\beta$-catenin pathway.

Nonetheless, it has been reported that $L G R 6$ acts as a tumor suppressor gene in colorectal cancer [16]. These findings may suggest that the functional control of $L G R 6$ is complex, and its role depends on the tumor type. The clinical significance and function of $L G R 6$ in ESCC has been rarely reported thus far, but on the basis of our current study, it seems to have a function as a CSC marker.

Therefore, the function of $L G R 6$ needs to be verified in cultured cells. It is particularly interesting as to whether $L G R 6$ is involved in tumor progression or tumor suppression. It is also necessary to search for differences between $L G R 6$ and $L G R 5$. Further analyses in additional cases are desired.

\section{Conclusions}

We have demonstrated that $L G R 6$ may be an important prognostic factor in ESCC. LGR6 may also be useful as a CSC marker and may be applicable in future cancer treatments.

\section{List Of Abbreviations}


ESCC, esophageal squamous cell carcinoma; LGR6, leucine-rich repeat-containing G-protein-coupled receptor 6; $\mathrm{HE}$, hematoxylin and eosin; OS, overall survival.

\section{Declarations}

\section{Ethics approval and consent to participate}

This study was approved by the ethics committee of Shinshu University School of Medicine (approval no. 4088). The requirement of informed consent was waived by the ethics committee of Shinshu University School of Medicine, and an opt-out method was used because of the retrospective design of the study. The investigation was conducted in compliance with the Helsinki Declaration.

\section{Consent for publication}

Not applicable.

\section{Availability of data and materials}

All data generated and analyzed in the current study are available from the corresponding author on reasonable request.

\section{Competing interests}

The authors declare that they have no competing interests.

\section{Funding}

This study was partially supported by the Hokuto Foundation for Bioscience (grant awarded to TU). The funding body had no role in the study design, collection, analysis, or interpretation of the data or manuscript writing.

\section{Authors' contributions}

TE participated in the design of the study, performed the pathological analysis, and drafted the manuscript. TU, SK, SA, and MI helped with the pathological analysis. TU performed the statistical analysis. TN and YK conducted the immunohistochemistry. TN and US examined the clinical data of cases. $\mathrm{HO}$ and TU critically revised the draft for important intellectual content.

\section{Acknowledgments}

We are grateful to Yukihoro Kobayashi, Masanobu Momose, Yasuyo Shimojo, Naoko Ogiwara, Akiko Inamura, Chitoshi Arai, Marina Nuno, Kanade Wakabayashi, and Naoko Yamaoka at Shinshu University Hospital for their excellent technical assistance. We thank H. Nikki March, PhD, from Edanz (https://jp.edanz.com/ac) for editing a draft of this manuscript. 


\section{References}

1. Hsu SY, Kudo M, Chen T, Nakabayashi K, Bhalla A, van der Spek PJet al. The three subfamilies of leucine-rich repeat-containing $G$ protein-coupled receptors (LGR): identification of LGR6 and LGR7 and the signaling mechanism for LGR7. Mol Endocrinol. 2000;14:1257-1271.

2. Snippert HJ, Haegebarth A, Kasper M, Jaks V, van Es JH, Barker Net al. Lgr6 marks stem cells in the hair follicle that generate all cell lineages of the skin. Science. 2010;327:1385-1389.

3. Ruiz EJ, Oeztuerk-Winder F, Ventura JJ A paracrine network regulates the cross-talk between human lung stem cells and the stroma. Nat Commun. 2014;5:3175.

4. Blaas L, Pucci F, Messal HA, Andersson AB, Josue Ruiz E, Gerling Met al. Lgr6 labels a rare population of mammary gland progenitor cells that are able to originate luminal mammary tumours. Nature cell biology. 2016;18:1346-1356.

5. Guinot A, Oeztuerk-Winder F, Ventura JJ miR-17-92/p38alpha Dysregulation Enhances Wnt Signaling and Selects Lgr6+ Cancer Stem-like Cells during Lung Adenocarcinoma Progression. Cancer Res. 2016;76:4012-4022.

6. Ruan X, Liu A, Zhong M, Wei J, Zhang W, Rong Yet al. Silencing LGR6 Attenuates Stemness and Chemoresistance via Inhibiting Wnt/beta-Catenin Signaling in Ovarian Cancer. Mol Ther Oncolytics. 2019;14:94-106.

7. Abnet CC, Arnold M, Wei WQ Epidemiology of Esophageal Squamous Cell Carcinoma. Gastroenterology. 2018;154:360-373.

8. Nakajima T, Uehara T, Iwaya M, Kobayashi Y, Maruyama Y, Ota H Characterization of LGR5 expression in poorly differentiated colorectal carcinoma with mismatch repair protein deficiency. BMC Cancer. 2020;20:319.

9. Chai T, Shen Z, Zhang Z, Chen S, Gao L, Zhang Pet al. LGR6 is a potential diagnostic and prognostic marker for esophageal squamous cell carcinoma. J Clin Lab Anal. 2020;34:e23121.

10. Morrison SJ, Spradling AC Stem cells and niches: mechanisms that promote stem cell maintenance throughout life. Cell. 2008;132:598-611.

11. Huang PY, Kandyba E, Jabouille A, Sjolund J, Kumar A, Halliwill Ket al. Lgr6 is a stem cell marker in mouse skin squamous cell carcinoma. Nat Genet. 2017;49:1624-1632.

12. Dzobo K, Thomford NE, Senthebane DA Targeting the Versatile Wnt/beta-Catenin Pathway in Cancer Biology and Therapeutics: From Concept to Actionable Strategy. OMICS. 2019;23:517-538.

13. Hao HX, Jiang X, Cong F Control of Wnt Receptor Turnover by R-spondin-ZNRF3/RNF43 Signaling Module and Its Dysregulation in Cancer. Cancers (Basel). 2016;8.

14. Haakonsen DL, Rape M Ubiquitin levels: the next target against gynecological cancers? J Clin Invest. 2017; 127:4228-4230.

15. Hauser AS, Attwood MM, Rask-Andersen M, Schioth HB, Gloriam DE Trends in GPCR drug discovery: new agents, targets and indications. Nat Rev Drug Discov. 2017;16:829-842. 
16. Gong X, Carmon KS, Lin Q, Thomas A, Yi J, Liu Q LGR6 is a high affinity receptor of R-spondins and potentially functions as a tumor suppressor. PLoS One. 2012;7:e37137.

\section{Figures}
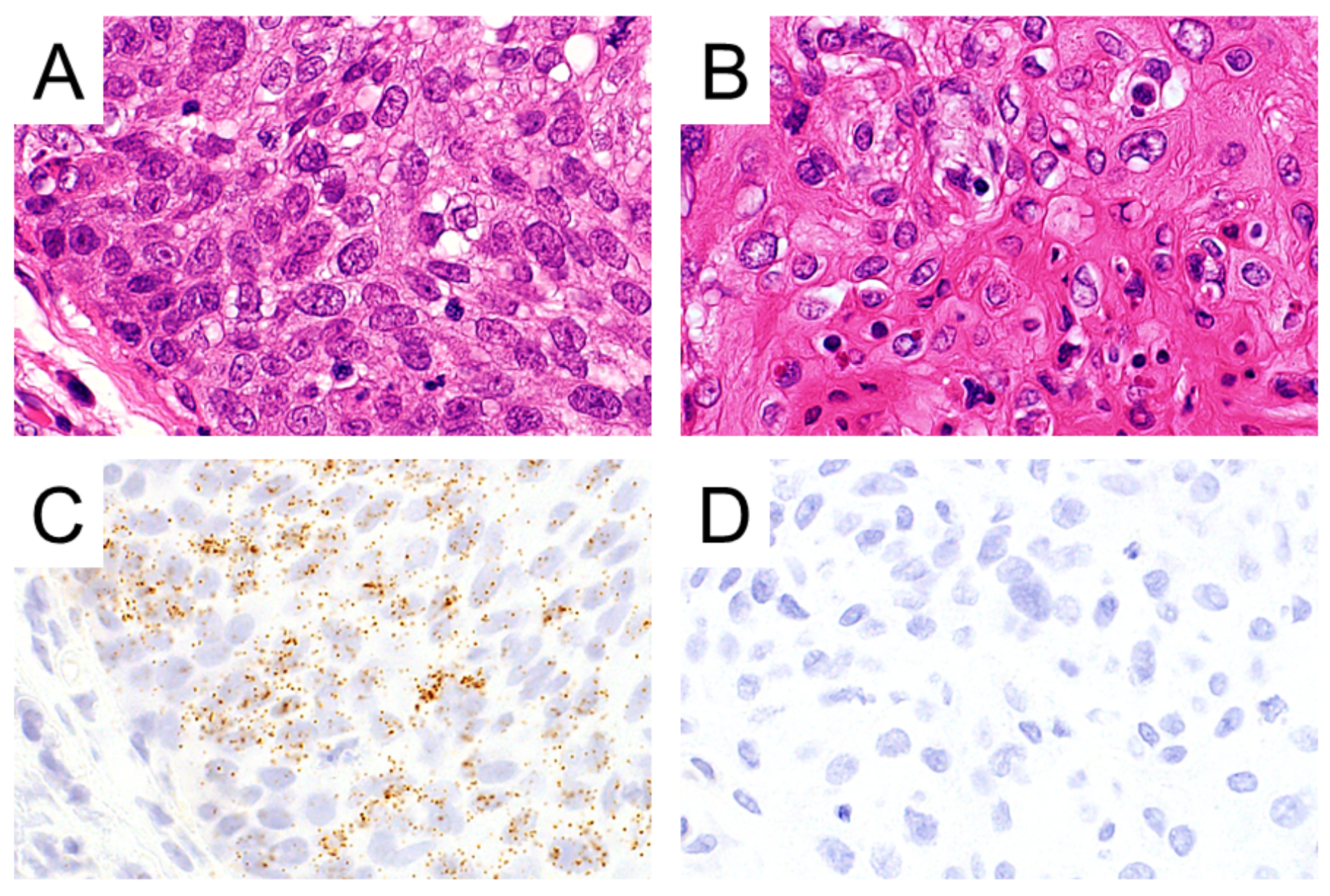

\section{Figure 1}

Representative images of LGR6. Representative features in cases with high LGR6 expression (A and C) and low LGR6 expression (B and D). (A and B, HE; C and D, LGR6). 


\section{- LGR6 - positive \\ -- LGR6 - negative}

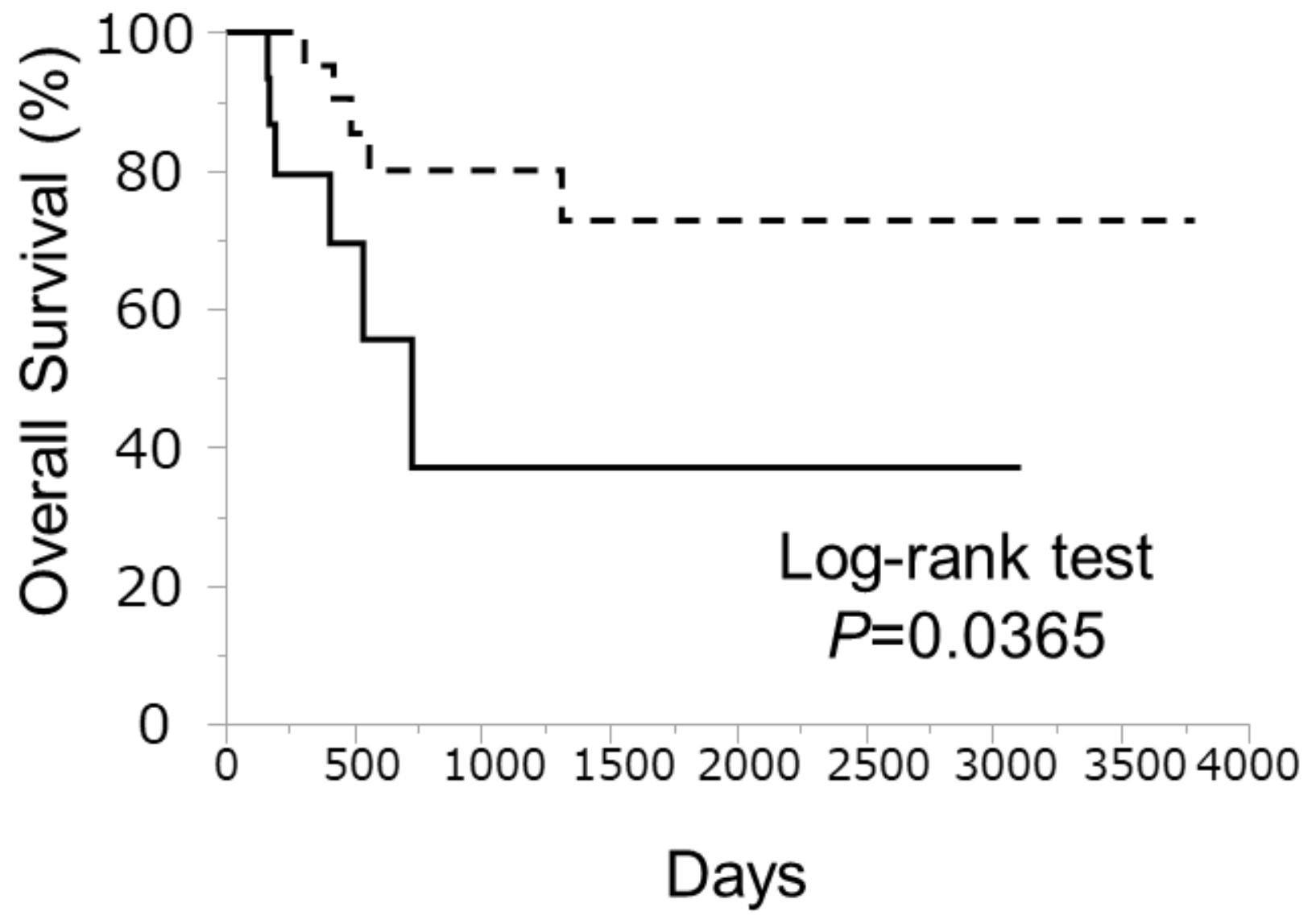

Figure 2

Prognostic value of LGR6 by Kaplan-Meier analysis. High LGR6 expression [median OS: 322 (range; 175-555.5) days] was associated with significantly worse OS than low LGR6 expression [median OS: 992 (range; 435.5-1825.5) days] (log-rank test, $P=0.0365$ ). 